\title{
Effect of cutting parameters on cutting temperature of TiAL6V4 alloy
}

\begin{abstract}
A Finite Element Modeling (FEM) and Simulation was Used to Investigate the Effect of Tool Rake Angle, Cutting Speed and Feed Rate on the Cutting Temperature of Tial6v4 Alloy. the Purpose of this Study was to Find Proper Cutting Parameters for Machining of Titanium Alloy where Cutting Temperature was Lowest. A FEM Based on ABAQUS Software which Involves Jonson-Cook Material Model and Coulomb's Friction Law was Applied to Simulate an Orthogonal Cutting Process. in this Simulation Work, a Range of Tool Rake Angle from $0^{\circ}$ to $10^{\circ}$, a Range of Cutting Speed from $300 \mathrm{~m} / \mathrm{min}$ to $600 \mathrm{~m} / \mathrm{min}$ and a Range of Feed Rate between $0.1 \mathrm{Rev} / \mathrm{mm}$ and $0.25 \mathrm{Rev} / \mathrm{mm}$ were Investigated. the Simulation Results Indicated that Increase in Rake Angle Reduces Cutting Temperature while Increasing Cutting Speed and Feed Rate Increase the Cutting Temperature.
\end{abstract}

Keyword: Finite element modeling; Rake angle; Titanium alloy 